\title{
Prenatal screening for cystic fibrosis: An early report card
}

\author{
Glenn E. Palomaki
}

The report by Dr. Strom and his colleagues at a large reference laboratory provides CFTR results from over 300,000 individuals tested during a 17 -month time period ending in December 2002. ${ }^{1}$ It illustrates just how common this testing has become in the last few years. Direct testing for cystic fibrosis became possible soon after the CFTR gene was cloned in 1988, but widespread prenatal screening did not occur until 2001, some 14 years later. This delay was due, in part, to the need for a relatively inexpensive and reliable DNA test technology that could detect a reasonable proportion of CFTR mutation carriers. Among non-Hispanic Caucasian and Ashkenazi Jewish individuals, a panel of 10 or fewer mutations was sufficient to identify about $80 \%$ of mutation carriers and, therefore, detect about $64 \%$ of carrier couples (or affected fetuses). By the mid 1990s, pilot trials in Europe and the United States demonstrated that DNA test technologies and programmatic aspects were suitable for introduction into practice. ${ }^{2}$ These reports included mutation frequencies in various racial/ethnic groups. At that time, however, professional guidelines suggested offering testing only to those with a family history. ${ }^{3}$ The next important step was taken in 1997 when an NIH Consensus Development Conference recommended that testing for cystic fibrosis be offered to couples seeking prenatal care. ${ }^{4}$ In 2001, the American College of Obstetricians and Gynecologists (ACOG) and the American College of Medical Genetics (ACMG) took further steps to standardize the process by recommending a minimum panel of 25 mutations, and promulgated model patient and provider information.,5 Although firm estimates are not available, the number of pregnancies screened in the United States grew from thousands of tests per year in 2000 to hundreds of thousands per year by 2003. One laboratory reported an increase from 1,000 tests per month in 2001 to 14,000 tests per month in mid-2003. ${ }^{7}$

According to data presented by Strom and his colleagues, 65 amniocenteses were performed when one (or both) parents carried only the $5 \mathrm{~T}$ polymorphism. These pregnancies, representing nearly $20 \%$ of the diagnostic testing performed, had a very low risk for having a fetus affected with cystic fibrosis. Such unwarranted invasive testing might be minimized by laboratories adhering to guidelines and by additional education efforts directed at both providers and patients. The ACMG Standards and Guidelines included recommendations that testing for the 5T/7T/9T polymor-

Foundation for Blood Research, Scarborough, Maine.

Glenn E. Palomaki, Director of Biometry, Foundation for Blood Research, PO Box 190, Scarborough, ME 04070-0190.

DOI: 10.1097/01.GIM.0000127272.60548.52 phism be performed reflexively only when the R117H mutation was identified. It was well known that only when the R117H mutation is found with the $5 \mathrm{~T}$ variant on the same chromosome is that mutation usually associated with classic cystic fibrosis. Unfortunately, Strom's laboratory (and some others) tested all samples for this relatively common polymorphism (approximately $5 \%$ of individuals are $5 \mathrm{~T}$ ). Systems need to be put in place to assure that implementation problems such as this are identified, quantified, and corrected.

An unanticipated problem occurred when one of the mutations that had not been subjected to evaluation during pilot trials was introduced directly into routine practice. The I148T mutation is known to occur about once in every 1000 genes among patients clinically affected with cystic fibrosis. When testing is performed in the general population, however, this mutation is found 100 times more often than expected. ${ }^{8}$ It is now thought that I148T is a polymorphism that is tightly linked to the actual disease-causing mutation 3199del6. Among the 90 couples submitting samples to Strom and his colleagues for diagnostic testing, five included one partner with only the I148T mutation identified. One compound heterozygote fetus was identified. Although this pregnancy was not terminated (follow-up at birth was not available), their experience demonstrates the types of potentially serious problems that can arise when pilot testing is not performed before routine use in practice.

The absence of data relating to overall program performance represents an important gap in this large laboratory's reported experience. Nichols Institute Quest Diagnostics, like most laboratories in the United States, implements screening using the "sequential" model. The partner is approached for testing only when the woman is found to carry a mutation, with the aim of identifying carrier couples whose pregnancies are at a 1 in 4 risk of developing classic cystic fibrosis. Based on clinical guidelines, these couples are then offered genetic counseling and consideration of prenatal diagnosis. The current experience reported by Strom and his colleagues shows that, in the current health care setting, it is nearly impossible to track the couple's progress using the sequential model. Many questions are left unanswered. ${ }^{9}$ How many pregnant women were tested, and was key information provided for proper interpretation (e.g., indication for testing, race/ethnicity, family history)? How many women were found to be carriers? How many of their partners were offered testing, and how many accepted? How many carrier couples were identified and what were their diagnostic and reproductive choices? There may be several reasons for this lack of information. In some instances, the information is not available, for example, the test request form does not ask the appropriate question(s), or the health care pro- 
vider does not supply the patient information when requested. In others, data collection is complicated by health care delivery systems. For example, when a woman is identified as a carrier, her partner's physician may routinely use another laboratory or direct his sample to another laboratory because of insurance coverage. If he were found to be a carrier, how does the process ensure that the two results reach the woman's prenatal care provider, that the couple's risk of having an affected child is reported, and that the couple is properly counseled?

The current ACOG/ACMG guidance for implementation does not acknowledge that prenatal screening for cystic fibrosis requires a programmatic approach to succeed despite the fact that all pilot trials utilized a programmatic approach. As evidence of this, the standards and guidelines do not address the expected proportion of carrier couples detected (essentially, the detection rate). Instead, they focus on the proportion of carrier individuals identified. Among the template reports and patient letters contained in the guidelines, there are no templates provided for a carrier couple (although a separate informational booklet is available for carrier couples). It is, therefore, not surprising that this laboratory, and most others in the United States, find it difficult to provide information about utilization or performance in practice, or to support the clinical utility associated with the CFTR testing they have performed.

Although Strom and his colleagues warn against cost analyses based on their data, some comment is warranted. If one assumes that the cost of laboratory testing is $\$ 150$ and that this represents the major component cost associated with a screening program (compared to other component costs such as diagnostic testing), approximately 50 million health care dollars were spent during the 17 months of testing. It is not known how many of the expected 312 carrier couples were actually found and informed of their pregnancy's risk, but only 90 carrier couples submitted amniotic fluid samples for diagnostic testing. Among those, 17 cases of cystic fibrosis were identified. Assuming that all were identified because of prenatal screening, the cost per case detected is about 3 million dollars; higher than projected by most previous economic analyses. ${ }^{10}$ This may be an overestimate because, as the authors suggest, additional cases may have been detected at other diagnostic laboratories. Regardless, there are insufficient data currently available from this large reference laboratory (that cannot track patient decision-making) to justify the expense of screening. If prenatal screening for cystic fibrosis is to be adequately assessed from a public health perspective, these data must be collected and evaluated in this type of setting.

What can be done to help document the clinical utility of current CFTR testing in the prenatal setting? First, methods to collect and evaluate data quantifying successes, and failures, must be developed, and the results used to improve the process. Issues to be addressed include utilization, quality, acceptability, and access. Secondly, the advantages and disadvantages of different testing approaches need to be reconsidered in the context of the realities of the U.S. health care delivery system. For example, potential issues raised about partner testing might be resolved by using the couple screening model. ${ }^{11}$ Another possibility is to work with health care payers to monitor implementation and find solutions to documented problems. For the sequential model, one solution might be to consider the couple, rather than the woman, as the testing unit. Thus, when a woman is found to be a carrier, contacting and testing the partner should be considered to be a part of the couple's testing sequence and be reimbursable (or the cost of testing a small proportion of partner's samples be included in the initial cost of screening). In addition, health care providers need to be encouraged by their professional organizations and screening laboratories to complete the requisition form entirely, as an aid to proper interpretation and to ensure that testing is offered to the appropriate population. Alternatively, laboratories can assist health care providers by working toward consistency in test ordering and reporting practices, keeping in mind the need for clear, simple, and standardized terminology. Policy makers should emphasize that the couple is the screening unit and urge laboratories to adopt an integrated programmatic approach to prenatal cystic fibrosis screening. This would be in keeping with other prenatal screening such as that for open neural tube defects or Down syndrome screening. In a short while, the window of opportunity for demonstrating the impact of prenatal screening for cystic fibrosis will begin to close. If these issues are not addressed, it is likely that significant gaps in knowledge will remain and hamper future public health decision-making.

\section{References}

1. Strom CM, Crossley B, Redman JB, Buller A, Quan F, Peng M et al. Cystic fibrosis screening: Lessons learned from the first 320,000 patients. Genet Med 2004;3: $136-141$.

2. Haddow JE, Bradley LA, Palomaki GE, Doherty RA, Bernhardt BA, Brock DJ et al. Issues in implementing prenatal screening for cystic fibrosis: results of a working conference. Genet Med 1999;1:129-135.

3. Statement of the American Society of Hum Genet on cystic fibrosis carrier screening. Am J Hum Genet 1992;51:1443-1444.

4. Genetic testing for cystic fibrosis. National Institutes of Health Consensus Development Conference Statement on genetic testing for cystic fibrosis. Arch Intern Med 1999;159:1529-1539.

5. Grody WW, Cutting GR, Klinger KW, Richards CS, Watson M, Desnick RJ. Laboratory standards and guidelines for population-based cystic fibrosis carrier screening. Genet Med 2001;3:149-154.

6. American College of Obstetrics and Gynecology and American College of Medical Genetics. Preconception and prenatal carrier screening for cystic fibrosis, clinical and laboratory guidelines, 2001. American College of Obstetrics and Gynecology publication, Washington, DC; October, 2001.

7. Vastag B. Cystic fibrosis gene testing a challenge: Experts say widespread use is creating unnecessary risks. JAMA 2003;290:2923-2924.

8. Rohlfs EM, Zhou Z, Sugarman EA, Heim RA, Pace RG, Knowles MR et al. The I148T CFTR allele occurs on multiple haplotypes: A complex allele is associated with cystic fibrosis. Genet Med 2002;4:319-323.

9. Bradley LA, Palomaki GE, Hyman D, Richards CS, Haddow JE. Prenatal cystic fibrosis (CF) screening: barriers to demonstrating clinical validity and utility in practice. ACMG Annual Clin Genet Meeting, March 2003, Abstract 164:88.

10. Haddow JE, Palomaki GE, Grosse S. Population-based prenatal screening for cystic fibrosis via carrier testing: ACCE review clinical utility, Question 35 and 36 - Financial Costs and Economic Benefits. Available at: http://www.cdc.gov/genomics/info/ reports/research/FBR/ACCE.htm. Accessed February 25, 2004.

11. Haddow JE. Prenatal screening for cystic fibrosis in the United States - time to re-evaluate implementation policies. J Med Screen 2003;10:105-106. 